\title{
Comment on "Using multiple observationally-based constraints to estimate climate sensitivity" by J. D. Annan and J. C. Hargreaves, Geophys. Res. Lett., 2006
}

\author{
S. V. Henriksson ${ }^{1}$, E. Arjas ${ }^{2}$, M. Laine ${ }^{1}$, J. Tamminen ${ }^{1}$, and A. Laaksonen ${ }^{1,3}$ \\ ${ }^{1}$ Finnish Meteorological Institute, 00101, Helsinki, Finland \\ ${ }^{2}$ Department of Mathematics and Statistics, University of Helsinki, 00014, Helsinki, Finland \\ ${ }^{3}$ Department of Physics, University of Kuopio, 70211, Kuopio, Finland
}

Received: 1 September 2009 - Published in Clim. Past Discuss.: 21 October 2009

Revised: 30 June 2010 - Accepted: 30 June 2010 - Published: 2 July 2010

\begin{abstract}
In their article from 2006, Annan and Hargreaves present a probabilistic estimate of climate sensitivity obtained by using Bayes' theorem to combine information from different sources. In this comment article we critisize two aspects of their reasoning, namely using probability density functions and likelihood functions interchangeably and the assumed independence of evidence from the different sources. The derivation of their result rests on key assumptions, some stated explicitly and some left implicit, which could be unrealistic. Thus their study does not convincingly reduce the large uncertainty of climate sensitivity remaining in previous observationally-based studies.
\end{abstract}

\section{Introduction}

In their work, Annan and Hargreaves (Annan and Hargreaves, 2006; hereinafter referred to as AH06) obtain an estimate for the uncertainty of climate sensitivity by combining three different sources of information. The estimate is presented in the form of a posterior (probability) density. In particular, the authors claim having derived the $95 \%$ Bayesian confidence (credible) interval $\left(1.7^{\circ} \mathrm{C}, 4.9^{\circ} \mathrm{C}\right)$ for the sensitivity parameter. This interval is very narrow in comparison to most observationally-based studies, in which the symmetric 90\% confidence interval can contain values of climate sensitivity greater than $10^{\circ} \mathrm{C}$ (Frame et al., 2005; Hegerl et al., 2007). Annan and Hargreaves use Bayes' Theorem to com-

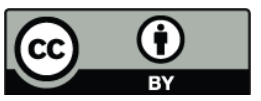

Correspondence to: S. V. Henriksson (svante.henriksson@fmi.fi) bine three sources of observational evidence, viz. estimates from the 20th century warming, volcanic forcing, and the last glacial maximum, into a single estimate of climate sensitivity. We believe that their conclusion is not warranted, as their derivation of the posterior distribution is based on several assumptions, which were left partly implicit, or which, if stated and considered critically, are unlikely to be realistic. The details of our argument are shown below.

\section{Bayesian inference}

Following the notation in AH06, Bayes' Theorem can be written in the form

$f(x \mid O, H)=f(O \mid x, H) f(x \mid H) / f(O \mid H)$,

where $x=$ the parameter to be estimated, $H=$ old data, $O=$ new data, and $f$ is a generic notation for conditional probability density functions. Thus this formula expresses how the posterior distribution $f(x \mid H)$ based on old data $H$ can be updated into the posterior $f(x \mid O, H)$ by also accounting for the new data $O$ and the likelihood function $f(O \mid x, H)$. In AH06 the authors apply Bayes' Theorem on the parameter $x=$ the climate sensitivity, and the data $H=$ 20th century warming, $O=\left(O_{1}, O_{2}\right)$ with $O_{1}=$ volcanic cooling, $\mathrm{O}_{2}=$ last glacial maximum. Then the crucial assumption is made that the three different sources of data, i.e., $H, O_{1}$ and $O_{2}$, are conditionally independent given the value of the sensitivity parameter $x$. Under this condition, Bayes' Theorem gets the form

$f\left(x \mid O_{1}, O_{2}, H\right)=f\left(O_{1} \mid x\right) f\left(O_{2} \mid x\right) f(x \mid H) / f\left(O_{1}, O_{2} \mid H\right)$.

Published by Copernicus Publications on behalf of the European Geosciences Union. 
Here the denominator does not depend on $x$ and can therefore be viewed as a proportionality constant. It becomes evident from going through the reasoning of AH06 that they use probability density functions (pdfs) and likelihood functions interchangeably, i.e., the likelihood functions $f\left(O_{1} \mid x\right)$, $f\left(\mathrm{O}_{2} \mid x\right)$ appearing in Bayes' Formula above are replaced by corresponding estimated pdfs $f\left(x \mid O_{1}\right)$ and $f\left(x \mid O_{2}\right)$, obtained in AH06 by considering the potential range of values $x$, then choosing three points that they believe would reasonably correspond to the 2.5 and 97.5 quantiles and the mode of $f\left(x \mid O_{1}\right)$, and similarly of $f\left(x \mid O_{2}\right)$, and finally fitting Gamma or Gaussian distributions to these points in order to obtain densities. The same method was used for deriving $f(x \mid H)$.

Two immediate observations can be made concerning this procedure: First, the quantiles and the mode are not based on proper Bayesian posterior densities $f\left(x \mid O_{1}\right), f\left(x \mid O_{2}\right)$ and $f(x \mid H)$ obtained from data by applying the strict rules of Bayesian statistical inference. Second, even if the densities $f\left(x \mid O_{1}\right)$ and $f\left(x \mid O_{2}\right)$ had been obtained by a proper Bayesian analysis and would thereby have the status of posterior density, they cannot be given the role of likelihood functions $f\left(O_{1} \mid x\right), f\left(O_{2} \mid x\right)$ unless a uniform prior density is assumed in the entire range of $x$. However, in this case it would be a very strange assumption, particularly in view of the fact that the above formula already involves the prior density $f(x \mid H)$, and uniform priors should then be assigned twice more to pdfs that do not originate from Bayesian analyses.

\section{Combining data from different sources}

In this section we point out a more fundamental problem in the approach of AH06 to combine the pdfs by the Bayesian method, viz. their assumption that the three different sources of data, $\mathrm{H}, \mathrm{O}_{1}$ and $\mathrm{O}_{2}$, are conditionally independent given the value of the sensitivity parameter $x$. We believe that this assumption is seriously flawed. To illustrate this, consider how estimates for climate sensitivity are derived from observational data. According to the simple heat balance equation (Andreae et al., 2005; Kiehl, 2007):

$c \mathrm{~d}(\Delta T) / \mathrm{d} t=\Delta Q-\lambda \Delta T$,

where $c$ is the ocean heat capacity per unit area, $\Delta T$ is the temperature change, $\Delta Q$ is the the total climate forcing due to natural and human factors and $\lambda$ is the climate feedback parameter. The climate sensitivity is defined as the equilibrium temperature change due to a doubling of carbon dioxide in the atmosphere, which means that the relation $\Delta T_{2 x \mathrm{CO}_{2}}=\Delta Q_{2 x \mathrm{CO}_{2}} / \lambda$ holds. The radiative forcing resulting from a doubling of the carbon dioxide concentration level is quite well known and given by $\Delta Q_{2 x \mathrm{CO}_{2}}=3.7 \mathrm{~W} / \mathrm{m}^{2}(\mathrm{Ra}-$ maswamy et al., 2001). Using the above relation, the climate sensitivity can be solved:

$\Delta T_{2 x \mathrm{CO}_{2}}=3.7 \Delta T /(\Delta Q-c \mathrm{~d}(\Delta T) / \mathrm{d} t)$.

When estimating the climate sensitivity parameter $x$ from observational data, uncertainty in the values of all three quantities $c, \Delta T$ and $\Delta Q$ contribute to the uncertainty in the derived estimate. Moreover, the latter two, $\Delta T$ and $\Delta Q$, even vary with time. A typical estimate for $c$ is 0.6 $1.6 \mathrm{GJ} \mathrm{m}^{-2} \mathrm{~K}^{-1}$ (Andreae et al., 2005; Levitus et al., 2000; Folland et al., 2001). Uncertainty in the value of $\Delta Q$ is large as well, the current estimate for anthropogenic $\Delta Q$ by the IPCC being $0.6-2.4 \mathrm{Wm}^{-2}$ (Forster et al., 2007). The temperature time series data involve various types of uncertainty and noise, which also contribute to the uncertainty of a climate sensivity estimate. In a proper Bayesian analysis and estimation of climate sensitivity, all these uncertainties in the values of the model parameters should be considered jointly, in terms of their joint probability distributions. The estimate of climate sensitivity would then be obtained by finally integrating out all other parameters from the resulting joint posterior.

Estimates of $c$ and $\Delta Q$ and their uncertainty in the references of AH06 can come, if not from the same source, then at least from similar reasoning and principles. References for all three different observational constraints use climate models in their reasoning (Andronova and Schlesinger, 2001; Knutti et al., 2002; Wigley et al., 2005; Annan et al., 2005) and it is very likely that the different models involve common sources of uncertainty. This concerns particularly estimates of the ocean heat capacity per unit area $c$ and radiative forcing $\Delta Q$. And in fact, the data from volcanic cooling used in AH06 does not include uncertainty of $\Delta Q$ at all (Wigley et al., 2005). Annan and Hargreaves discuss the concern of similar biases in climate models at the end of Sect. 3.4., and exclude two additional lines of evidence from their main conclusion partly because of this, but make no argument as to why the concern would not undermine the conditional independence assumption for the three lines of evidence used. For the last glacial maximum constraint, uncertainty in the ocean heat capacity per unit area $c$ might not effect the end result as the glacial climate had enough time to reach equilibrium. Also radiative forcing estimates at the LGM might be considered more independent from 20th century estimates than for the volcanic cooling case, but dependency might arise for instance from dust forcing. Dust radiative forcing affects estimates from 20th century warming and the LGM in opposite ways. Increasing a dust forcing estimate increases the corresponding climate sensitivity estimate from 20 th century warming and decreases the corresponding climate sensitivity estimate from the LGM. If the dust forcing estimates are correlated, the forcing estimates together with the prior and the likelihood determine how they affect the width of the posterior. With suitable assumptions for dependencies between data a very wide range of results are possible, in the 
case of the data used in AH06 in theory even a narrower result than the one obtained there.

For the LGM constraint, also uncertainty in the climate and forcing data and difference between glacial and present climate remain challenges for using the constraint in estimates for the climate sensitivity. In AH06 the former is treated by adding an independent Gaussian error to the pdf and the latter rather superficially without any systematic methodology. The LGM constraint in AH06 is much tighter than the 20th century warming constraint and therefore dominates the end result, although the climate and radiative forcing information has a lot of uncertainty (Hegerl et al., 2007) and although the 20th century conditions are more similar to and relevant for future climate predictions. As we are not aware of any totally observationally-based study (or one not using a general circulation model) of the LGM presenting a pdf for climate sensitivity as a result it is also hard to pinpoint dependencies or the lack thereof between 20th century warming and LGM data.

\section{Sensitivity analysis}

In order to come up with rough estimates of the quantitative effect, which the assumptions made in AH06 had on its end result, we performed two sensitivity calculations by changing or relaxing these assumptions. Consider first the effect of ignoring the volcanic cooling constraint. This sensitivity calculation is highly relevant as the volcanic cooling data used do not take radiative forcing uncertainty into account at all and dependency between the volcanic cooling and 20th century warming data through ocean heat uptake is not addressed either. Ignoring the volcanic cooling constraint but keeping all other assumptions untouched increases the upper bound of the $95 \%$ confidence interval to 5.6 degrees, as is illustrated in Fig. 1. In our second calculation we further widen both bounds of the $95 \%$ confidence interval in the LGM estimate by half a degree, thereby increasing the standard deviation of the corresponding Gaussian distribution from 1.7 degrees to 2 degrees. A difference of this kind could arise from a small change in the uncertainty in any of the factors' contribution to the uncertainty in the LGM estimate in AH06, and possibly also from taking into account dependency between the data from 20th century warming and LGM cooling. This sensitivity calculation is also relevant as the distribution for LGM cooling in AH06 is not produced by a systematic study, nor is there any argument in support of independence of e.g. dust radiative forcing in these two cases.

In a subsequent article, Annan and Hargreaves present newer calculations on the pdf of climate sensitivity and reach even narrower 95\% confidence intervals than in AH06 (Annan and Hargreaves, 2009; hereinafter referred to as AH09). They recommend the IPCC to increase the level of confidence (a probability assessment) in that the climate sensitivity would be at most 4.5 degrees, to $95 \%$. However, the

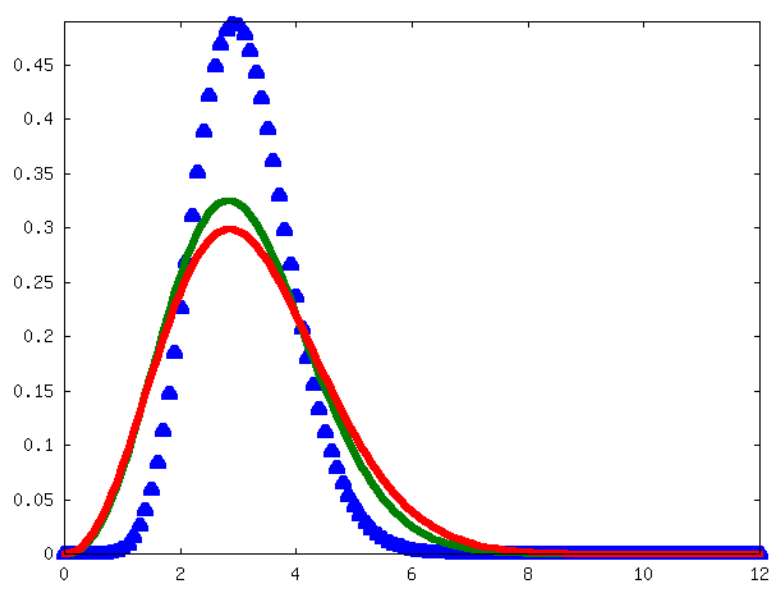

Fig. 1. Pdf for climate sensitivity (in degrees Celsius) from AH06 (blue triangles), pdf obtained by ignoring the volcanic cooling constraint (green solid line) and pdf obtained by ignoring the volcanic cooling constraint and adding 0.5 degrees to the upper and lower bound of the $95 \%$ confidence level in the pdf corresponding to the LGM (red solid line).

results in AH09 are achieved by using narrower priors, again taken into use without sufficient support. In particular, anticipated economic damages caused by climate change cannot be used as arguments for estimation of the prior. We cannot perceive that there could be meaningful estimates of economic damages caused by climate change, other than what could be obtained by first estimating the size of the climate change itself. Our main concerns relating to AH06 thus remain unanswered also in AH09.

\section{Conclusions}

The original idea of AH06, to combine information from different sources and thereby arrive at an improved estimate for the climate sensitivity, is interesting and in principle achievable by applying the tools provided by Bayesian statistical inference. However, very careful attention should be paid to assumptions made as they have a strong impact on the result. Annan and Hargreaves are carrying out their derivation by assuming, implicitly, that the climate sensitivity pdfs from different lines of evidence originate from Bayesian analyses and then applying uniform priors to them afterhand, and explicitly, that the different lines of evidence are conditionally independent given the climate sensitivity. The first assumption is clearly not adequate, and the second one can also be invalid, even to a good approximation, as the different results involve common uncertainties. These concerns make the reliability of the resulting pdf highly questionable. Therefore high values of climate sensitivity cannot be ruled out in the way done by Annan and Hargreaves. 
Acknowledgements. Svante Henriksson gratefully acknowledges funding from the Academy of Finland, project no. 129355. The financial support by the Academy of Finland Centre of Excellence program (project no. 1118615) is gratefully acknowledged. We thank James Annan for pointing out a typo and an inexact citation in an earlier version of the manuscript.

Edited by: V. Brovkin

\section{References}

Andreae, M. O., Jones, C. D., and Cox, P. M.: Strong present-day aerosol cooling implies a hot future, Nature, 435, 1187-1190, 2005.

Andronova, N. G. and Schlesinger, M. E.: Objective estimation of the probability density function for climate sensitivity, J. Geophys. Res., 106, 22605-22611, doi:10.1029/2000JD000259, 2001.

Annan, J. D., Hargreaves, J. C., Ohgaito, R., Abe-Ouchi, A., and Emori, S.: Efficiently constraining climate sensitivity with paleoclimate simulations, SOLA, 1, 181-184, 2005.

Annan, J. D. and Hargreaves, J. C.: Using multiple observationallybased constraints to estimate climate sensitivity, Geophys. Res. Lett., 33, L06704, doi:10.1029/2005GL025259, 2006.

Annan, J. D. and Hargreaves, J. C.: On the generation and interpretation of probabilistic estimates of climate sensitivity, Climatic Change, doi:10.1007/s10584-009-9715-y, 2009.

Folland, C. K., Rayner, N. A., Brown, S. J., Smith, T. M., Shen, S. S. P., Parker, D. E., Macadam, I., Jones, P. D., Jones, R. N., Nicholls, N., and Sexton D. M. H.: Global temperature change and its uncertainties since 1861, Geophys. Res. Lett., 28, 26212624, doi:10.1029/2001GL012877, 2001.

Forster, P., Ramaswamy, V., Artaxo, P., Berntsen, T., Betts, R., Fahey, D. W., Haywood, J., Lean, J., Lowe, D. C., Myhre, G., Nganga, J., Prinn, R., Raga, G., Schulz, M., and Van Dorland, R.: Changes in Atmospheric Constituents and in Radiative Forcing, in: Climate Change 2007: The Physical Science Basis. Contribution of Working Group I to the Fourth Assessment Report of the Intergovernmental Panel on Climate Change, edited by: Solomon, S., Qin, D., Manning, M., Chen, Z., Marquis, M., Averyt, K. B., Tignor, M., and Miller, H. L., Cambridge University Press, Cambridge, UK and New York, NY, USA, 2007.
Frame, D. J., Booth, B. B. B., Kettlebrough, J. A., Stainforth, D. A., Gregory, J. M., Collins, M., and Allen, M. R.: Constraining climate forecasts: The role of prior assumptions, Geophys. Res. Lett., 32, L090702, doi:10.1029/2004GL022241, 2005.

Hegerl, G. C., Zwiers, F. W., Braconnot, P., Gillett, N. P., Luo, Y., Marengo Orsini, J. A., Nicholls, N., Penner, J. E., and Stott, P. A.: Understanding and Attributing Climate Change, in: Climate Change 2007: The Physical Science Basis. Contribution of Working Group I to the Fourth Assessment Report of the Intergovernmental Panel on Climate Change, edited by: Solomon, S., Qin, D., Manning, M., Chen, Z., Marquis, M., Averyt, K. B., Tignor, M., and Miller, H. L., Cambridge University Press, Cambridge, UK and New York, NY, USA, 2007.

Kiehl, J. T.: Twentieth century climate model response and climate sensitivity, Geophys. Res. Lett., 34, L22710, doi:10.1029/2007GL031383, 2007.

Knutti, R., Stocker, T. F., Joos, F., and Plattner, G.-K.: Constraints on radiative forcing and future climate change from observations and climate model ensembles, Nature, 416, 716-723, 2002.

Levitus, S., Antonov, J. I., Boyer, T. P., and Stephens, C.: Warming of the world ocean, Science, 287, 2225-2229, 2000.

Ramaswamy, V., Boucher, O., Haigh, J., Hauglustaire, D., Haywood, J., Myhre, G., Nakajima, T., Shi, G.-Y., and Solomon, S.: Radiative forcing of climate change, in: Climate Change 2001: The Scientific Basis. Contribution of Working Group I to the Third Assessment Report of the Intergovernmental Panel on Climate Change, Cambridge University Press, Cambridge, UK and New York, NY, USA, 349-416, 2001.

Wigley, T. M. L., Amman, C. M., Santer, B. D., and Raper, S. P.: Effect of climate sensitivity on the response to volcanic forcing, $\mathrm{J}$. Geophys. Res., 110, D09107, doi:10.1029/2004JD005557, 2005. 